\title{
Mental health problems, needs, and service use among people living within and outside Wuhan during the COVID-19 epidemic in China
}

\author{
Bao-Liang Zhong ${ }^{1,2,3 \#}$, De-Yi Zhou ${ }^{4 \#}$, Min-Fu He ${ }^{5}$, Yi Li ${ }^{1,2,3}$, Wen-Tian Li $^{1,2,3}$, Chee H. Ng \\ Yu-Tao Xiang ${ }^{7,8}$, Helen Fung-Kum Chiu' ${ }^{9}$
}

${ }^{1}$ Department of Psychiatry, Wuhan Mental Health Center, Wuhan, China; ${ }^{2}$ Affiliated Wuhan Mental Health Center, Tongji Medical College of Huazhong University of Science \& Technology, Wuhan, China; ${ }^{3}$ Research Center for Psychological and Health Sciences, China University of Geosciences, Wuhan, China; ${ }^{4}$ Department of Psychiatry, The Affiliated Wuxi Mental Health Center of Nanjing Medical University, Wuxi, China; ${ }^{5}$ Department of Social Medicine and Health Management, School of Public Health, Jilin University, Changchun, China; ${ }^{6}$ Department of Psychiatry, The Melbourne Clinic and St Vincent's Hospital, University of Melbourne, Richmond, Victoria, Australia; ${ }^{7}$ Unit of Psychiatry, Institute of Translational Medicine, Faculty of Health Sciences, University of Macau, Macao SAR, China; ${ }^{8}$ Center for Cognition and Brain Sciences, University of Macau, Macao, China; ${ }^{9}$ Department of Psychiatry, The Chinese University of Hong Kong, Hong Kong, China

Contributions: (I) Conception and design: BL Zhong, WT Li, Y Li, YT Xiang; (II) Administrative support: WT Li, Y Li; (III) Provision of study materials or patients: BL Zhong, DY Zhou; (IV) Collection and assembly of data: BL Zhong, DY Zhou; (V) Data analysis and interpretation: BL Zhong, DY Zhou, MF He, CH Ng, YT Xiang, HFK Chiu; (VI) Manuscript writing: All authors; (VII) Final approval of manuscript: All authors.

\#These authors contributed equally to this work.

Correspondence to: Prof. Yi Li, MD, PhD; Prof. Wen-Tian Li, MD, PhD. No. 89, Gongnongbing Rd., Wuhan Mental Health Center, Jiang'an District, Wuhan 430012, China. Email: psylee@163.com; damoguyan933@163.com. Prof. Yu-Tao Xiang, MD, PhD. 3/F, Building E12, Faculty of Health Sciences, University of Macau, Avenida da Universidade, Taipa, Macau SAR, China. Email: xyutly@gmail.com.

Background: Crisis mental health services for the general population are an essential component in combating the COVID-19 epidemic. To facilitate planning of mental health services, empirical data on mental health problems and service utilization of populations affected by the epidemic are urgently needed. This study investigated the prevalence of mental health problems of residents within and outside Wuhan, China, as well as their patterns of mental health service utilization.

Methods: From January 27 to February 2, 2020, an online cross-sectional survey recruited residents from 4 subpopulations: Wuhan residents ( $\mathrm{n}=2,617)$, migrants from Wuhan (those who left Wuhan before lockdown, $\mathrm{n}=930$ ), other Hubei residents ( $\mathrm{n}=633$ ), and residents of other provinces of China ("other residents", $\mathrm{n}=3,561$ ). Participants' mental health problems and patterns of utilization of mental health services in recent days were assessed with standardized assessments.

Results: Among the 4 groups, Wuhan residents had the highest rate of any type of mental health problem (i.e., psychological distress: $41.9 \%$ ), followed by migrants from Wuhan (34.2\%), other Hubei residents (24.8\%), and other residents $(21.1 \%)$. Rates of perceived mental health needs and utilization of mental health services during recent days were $10.2 \%$ and $1.9 \%$ for Wuhan residents, $8.4 \%$ and $1.8 \%$ for migrants from Wuhan, $6.2 \%$ and $1.1 \%$ for other Hubei residents, and $6.4 \%$ and $1.0 \%$ for other residents, respectively. Overall, 63.0\% mental health service users received services via internet and telephone, and $83.1 \%$ participants with perceived mental health needs ascribed their lack of help-seeking to barriers to accessibility and availability.

Conclusions: The COVID-19 epidemic is associated with unprecedented levels of mental health problems and needs in affected residents. To address the unmet mental health needs, internet- and telephone-based mental health services should be widely provided to residents, especially those in the worst-affected regions. 
Keywords: Mental health problems; mental health services; general population; epicenter; COVID-19

Submitted May 21, 2020. Accepted for publication Sep 03, 2020.

doi: 10.21037/atm-20-4145

View this article at: http://dx.doi.org/10.21037/atm-20-4145

\section{Introduction}

At the end of 2019, the outbreak of a respiratory illness caused by the Severe Acute Respiratory Syndrome Coronavirus 2 (SARS-CoV-2), now named Coronavirus Disease 2019 (COVID-19), was first reported in Wuhan, Hubei province, which is also the largest city in central China with a total population of 14 million. The situation rapidly worsened on January 20, 2020, as the number of confirmed COVID-19 cases increased to 198 with 3 deaths in Wuhan and human-to-human transmission of SARS$\mathrm{CoV}-2$ was clearly confirmed $(1,2)$.

To control the rapid spread of SARS-CoV-2, the Wuhan government locked down the whole city on January 23, and unprecedented measures were adopted: stopping all public transportations, closing all public spaces, banning any public gathering, and urging the residents to stay indoors (2). Nevertheless, an estimated 5 million persons had left Wuhan to other parts of China for Lunar New Year celebrations before the lockdown (3). Later, it became clear that the SARS-CoV-2 had spread to Wuhan's surrounding cities in Hubei, other provinces in China, and many other countries. As of May 18, the COVID-19 epidemic had caused 4,645 deaths from 84,494 infected persons in China, and 307,202 deaths from 4,534,327 infected persons in over 200 other countries/territories (4). Wuhan and other parts of Hubei province were the worst affected regions in China: both regions accounted for $59.6 \%$ and $21.1 \%$ of the total infections, and $83.3 \%$ and $13.8 \%$ of the total deaths in China, respectively (5). By January 27, the lockdown had expanded to the whole province of Hubei and approximately 60 million inhabitants lived in quarantine. In response to this serious situation, all the 31 provinces of China had launched the "Top-level Response to Major Public Health Emergencies" by January 29. Nationwide measures were subsequently taken to prevent the spread of COVID-19, including isolating and treating imported cases from Wuhan, extension of the Spring Festival holiday, traffic controls, and cancellation of mass gathering activities (6). Due to the rapid worldwide spread of COVID-19, the World Health Organization (WHO) declared the
COVID-19 outbreak a "public health emergency of international concern" on January 30 and finally as a pandemic on March $11(7,8)$.

The epidemic of infectious diseases, however, also has an adverse impact on the mental health of the general public (9). For example, in 2003, the SARS outbreak caused considerable fear and panic in the general population, especially in seriously affected cities during its early stage when there were large increases in number of infections and deaths (10-13). Nevertheless, compared to SARS, the COVID-19 pandemic presents a more profound negative impact on the mental health of the general population due to the higher numbers of infected cases, deaths, and affected countries globally. Unlike the previous SARS epidemic, the COVID-19 outbreak is occurring concurrently with 'infodemic' where misinformation and rumors can be easily and immediately transmitted via social media, which can further exacerbate mental health problems in people affected by the epidemic (14). Moreover, although the mass quarantine in Wuhan is necessary and effective for delaying the transmission of SARS-CoV-2 (15), studies have found that quarantine is associated with wide-ranging and substantial mental health problems such as psychological distress, anxiety, sadness, fear, and post-traumatic stress symptoms (16). The combined effects of COVID-19 epidemic and mass quarantine would likely cause concern regarding the deteriorating mental health of the population, particularly those living in the epicenter, Wuhan, and its surrounding regions in Hubei.

Psychological crisis intervention is an essential component of infectious disease prevention and control measures. To address the mental health problems caused by the COVID-19 epidemic, on January 27, the National Health Commission of China (NHCC) released the "Principles for Emergency Psychological Crisis Intervention for SARS-CoV-2 Pneumonia Epidemic" (17). These principles recommend that mental health workers should identify persons at risk for psychological crisis by implementing detailed and dynamic mental health assessment and provide appropriate psychological interventions to those in need according to their risk 
level of poor mental health. However, due to the lack of empirical data on the risk of mental health problems and needs of the population in the context of the COVID-19 epidemic, it remains unknown how best to provide mental health services to those in need.

At individual level, timely mental health services are urgently needed for various people affected by the COVID-19 epidemic (18). As a case in point, since the COVID-19 outbreak, the psychological hotline of Wuhan Mental Health Center, Hubei's largest public mental health care provider, had been overwhelmed with an average of over 1,000 calls per day, far exceeding its usual capacity (20-50 calls per day). Most calls were made by residents in Wuhan and other parts of Hubei province (>60\%), while others were from Wuhan residents who had traveled to other places (10-20\%). To facilitate the planning and development of appropriate mental health services during the COVID-19 epidemic, this study investigated the prevalence of mental health problems of residents within and outside Wuhan, China, as well as their patterns of mental health service utilization. We present the following article in accordance with the SURGE reporting checklist (available at http://dx.doi.org/10.21037/atm-20-4145).

\section{Methods}

\section{Participants}

This cross-sectional online survey was conducted in 2020 between January 27, the day when Hubei province was locked down, and February 2. This was a period when the COVID-19 epidemic was rapidly spreading and cases were rising exponentially in China (January 10-February 5) $(6,19)$. Due to logistical difficulties in conducting a population-based sampling survey and the urgent need for epidemiological and health service data, recruitment during this unique period was done via selective networks of people living in Wuhan and other cities of Hubei province. A one-page invitation letter was used to facilitate the online recruitment of participants, which described the background, significance, procedures, anonymity, and voluntary participation of the survey, as well as the link and quick response $(\mathrm{QR})$ code of our online questionnaire. Local people disseminated the invitation letter among their social networks, and posted the letter to moments and groups of their Wechat (similar to "WhatsApp") and Weibo (similar to "Twitter") accounts. In addition, some local residents' committees and popular media outlets such as Yangtze River Daily and ChuTian Metropolis Daily also circulated the invitation letter.

Chinese people who were 16 years and onwards and willing to take part in the study were instructed to click the link or scan the QR code. After that, a binary question requested the respondents to confirm their willingness to participate. Those who endorsed this question were directed to complete the questionnaire. There was no restriction on participants' place of residence. Participants were asked whether they were working, studying, or living in Wuhan during the 2 months before the survey, and where they were currently living in. They were then divided into 4 mutually exclusive subpopulations: Wuhan residents (those who were currently living in Wuhan), migrants from Wuhan (those who were previously living in Wuhan, and currently living in parts of China other than Wuhan), other Hubei residents (those who were currently living in other parts of Hubei and not in Wuhan before), and residents of other provinces of China ("other residents" hereafter, those who were currently living in other provinces of China and not in Wuhan before).

The study was conducted in accordance with the Declaration of Helsinki (as revised in 2013). Before the survey, the Institutional Review Board of Wuhan Mental Health Center approved the survey protocol and the above-mentioned procedures of informed consent (No.: KY2020.01.01).

\section{Assessments}

The questionnaire consisted of 4 parts: demographics, experience and perceptions of COVID-19 epidemic, mental health problems, and mental health needs and service utilization. Demographic data included age, gender, marital status, employment, and education status.

Questions used for assessing the perceived severity of the epidemic in their current residence were: "How severe do you feel the COVID-19 epidemic is in the city/county of your current residence?" (severe vs. not severe), "Do you have any family member or close relative infected with SARS-CoV-2?" (yes vs. no), "Do you have any colleague, friend or classmate infected with SARS-CoV-2?" (yes vs. no), and "Do you have any neighbor of your community/ village infected with SARS-CoV-2?" (yes vs. no).

Mental health problems included psychological distress, anxiety symptoms, and depressive symptoms. Psychological distress over the past few weeks was assessed with the 12-item General Health Questionnaire (GHQ-12), which 
has been used in a wide variety of clinical and non-clinical settings (20,21). The scale consists of 6 positive and 6 negative items with each being rated on a dichotomous scale: $0=$ better than usual, $0=$ same as usual, $1=$ worse than usual, and $1=$ much worse than usual for positive items, and $0=$ not at all, $0=$ same as usual, $1=$ worse than usual, and $1=$ much worse than usual for negative items. The total score of GHQ-12 is the sum of the 12 items, ranging from 0 to 12 , with higher scores suggesting more severe psychological distress. The Chinese GHQ-12 is reliable and valid in Chinese population with a cut-off score of 3 or more indicating the presence of psychological distress $(22,23)$.

We used the validated Chinese 7-item Generalized Anxiety Disorder Scale (GADS-7) and 9-item Patient Health Questionnaire (PHQ-9) to assess the severity of anxiety and depressive symptoms over the last 2 weeks, respectively (24-26). All items of the 2 scales were answered on a 4 -point scale: $0=$ not at all, $1=$ several days, $2=$ over half the days, and $3=$ nearly every day. The total scores of the GADS-7 and PHQ-9 range from 0 to 21 and from 0 to 27, with a GADS-7 score of 6 or higher and a PHQ-9 score of 7 or higher denoting clinically significant anxiety and depressive symptoms, respectively $(24,27,28)$.

The question used for assessing residents' perceived mental health needs: "During recent days, did you recognize that you need professional help from mental health specialists because of your high level of stress, bad emotions, poor sleep, or other mental health problems? These specialists include psychiatrists, psycho-therapists, and psychological counselors." Respondents who endorsed a need for mental health care were asked whether they had used mental health services: "Did you seek any help from mental health specialists for your mental health problems?". Those who used mental health services were further asked about the type of interaction with mental health specialists: face-to-face visit at a psychiatric, psychological, or medical institution; psychological consultation and therapy via internet such as website and mobile application; and psychological consultation and therapy via telephone such as hotline, and others. Participants who reported a need for mental health care but did not use mental health services were asked to describe their main reasons for not using mental health services. Based on previous studies $(29,30)$, the barriers to care were classified into 3 categories: accessibility (cost, transportation, and competing personal/ family responsibilities), availability (lack of knowledge about service availability and services unavailable at the time required or in current residence place), and acceptability (attitudes towards mental disorders and mental health specialists).

\section{Statistical analysis}

Rates of prevalence of mental health problems, perceived mental health needs, and use of mental health services according to subpopulation were described. The rates, demographics and experience of the COVID-19 epidemic across the 4 subpopulations were compared using Chisquare test. The relative risks of mental health problems of Wuhan residents, migrants from Wuhan, and other Hubei residents in comparison to other residents were further examined with multiple logistic regression analyses. The presence of mental health problems was entered as the outcome variables, and subpopulation as the predictor of interest (reference category: other residents), adjusting for sets of covariates hierarchically. Covariates included demographics and characteristics of the COVID-19 epidemic. The relative odds of perceived mental health needs and use of mental health services of Wuhan residents, migrants from Wuhan, and other Hubei residents relative to other residents were tested in the same step-by-step manner. We used odds ratios (ORs) and their corresponding $95 \%$ confidence intervals (CIs) to quantify associations between outcome variables and the type of subpopulation. To facilitate the explanations of such associations, we adjusted for covariates step-by-step in multiple logistic regression analyses, for example, if the significant association of depression with Wuhan residents (versus other residents) became insignificant after adjusting for experience of the COVID-19 epidemic, we would speculate that subpopulation difference in the risk of depression is caused by different influence of the COVID-19 epidemic. The statistical significance level was set at $\mathrm{P}<0.05$ (twosided). SPSS software version 14.0 package (SPSS Inc., Chicago, Illinois, United States) was used for all analyses.

\section{Results}

Among the 7,750 Chinese residents who completed the online questionnaire, 9 were excluded due to infection with SARS-CoV-2. The final study sample included was 7,741 participants with an average age of 33.1 years [standard deviation (SD): 10.5 , range, $16-87$ ] and $32.8 \%$ being males. The sample consisted of 2,617 Wuhan residents, 930 migrants from Wuhan, 633 other Hubei residents, and 3,561 
other residents.

Detailed demographics and COVID-19 epidemic experience of the total sample and the 4 subpopulations are shown in Table 1. All demographic variables and the COVID-19 epidemic experience significantly differed across the groups $(\mathrm{P}<0.001)$.

Prevalence of mental health problems significantly differed across the groups $(\mathrm{P}<0.001)$, with the highest rate in Wuhan residents, followed by migrants from Wuhan, other Hubei residents, and other residents: psychological distress: $41.9 \%$ vs. $34.2 \%$ vs. $24.8 \%$ vs. $21.1 \%$, anxiety symptoms: $32.3 \%$ vs. $27.5 \%$ vs. $19.9 \%$ vs. $16.8 \%$, and depressive symptoms: $28.6 \%$ vs. $24.3 \%$ vs. $17.7 \%$ vs. $16.1 \%$ (Table 1). ORs of Wuhan residents, migrants from Wuhan, and other Hubei residents relative to other residents having mental health problems increased after controlling for demographic variables in multiple logistic regression analyses: psychological distress: $2.99(\mathrm{P}<0.001)$, $2.33(\mathrm{P}<0.001)$, and $1.47(\mathrm{P}=0.036)$, anxiety symptoms: $2.59(\mathrm{P}<0.001), 2.16(\mathrm{P}<0.001)$, and $1.45(\mathrm{P}=0.001)$, and depressive symptoms: $2.32(\mathrm{P}<0.001), 1.75(\mathrm{P}<0.001)$, and $1.29(\mathrm{P}=0.030)$. After adjusting for demographics and the COVID-19 epidemic variables, these OR estimates attenuated but remained statistically significant except that of depression among other Hubei residents (Table 2). Results of multiple logistic regression analyses that included demographics and the COVID-19 epidemic variables simultaneously show that, overall, respondents experiencing severe COVID-19 epidemic, having infected family members or close relatives, having infected colleagues, friends, or classmates, and having infected neighbors were at greater risk for psychological distress, anxiety symptoms, and depressive symptoms than their counterparts without these attributes (Table S1).

In total, 10.2\% Wuhan residents perceived a need for mental health care during recent days, but only $1.9 \%$ had sought help from mental health specialists. The respective corresponding rates were $8.4 \%$ and $1.8 \%$ for migrants from Wuhan, $6.2 \%$ and $1.1 \%$ for other Hubei residents, and $6.4 \%$ and $1.0 \%$ for other residents. Taken these rates together, $8.4 \%$ Wuhan residents, $6.6 \%$ migrants from Wuhan, $5.1 \%$ other Hubei residents, and $5.4 \%$ other residents had unmet mental health needs (Table 1). The significantly ORs of perceived need for mental health care in Wuhan residents and migrants from Wuhan compared to other residents remained statistically significant after adjusting for demographics and the COVID-19 epidemic variables (OR: 1.33, $\mathrm{P}=0.025$; $\mathrm{OR}: 1.34, \mathrm{P}=0.037)$ but became insignificant after additionally adjusting for mental health problems. The significantly higher ORs of mental health service use in Wuhan residents relative to other residents remained statistically significant after adjusting for demographic variables (OR: 1.93, $\mathrm{P}=0.004)$ but was insignificant in the subsequent adjustment analyses that included demographics, the COVID-19 epidemic variables, and mental health problems (Table 3). Results of multiple logistic regression analyses that included all demographics, COVID-19 epidemic variables, and mental health problems show that, some COVID-19 epidemic variables and most mental health problems were significantly associated with perceived mental health needs and service utilization of the residents (Table S2).

Table 4 illustrates type of care from specialists among those who utilized mental health services. In total, $63.0 \%$ users used remote mental health services (internet- and telephone-based). The corresponding rates were $77.5 \%$ in Wuhan residents, $58.8 \%$ in migrants from Wuhan, $85.8 \%$ in other Hubei residents, and $40.0 \%$ in other residents.

As shown in Table 5, among those with unmet mental health needs, $83.1 \%$ ascribed their lack of help-seeking from mental health professionals to barriers due to poor accessibility and availability. The corresponding rates were $85.4 \%$ in Wuhan residents, $91.8 \%$ in migrants from Wuhan, $68.8 \%$ in other Hubei residents, and $56.4 \%$ in other residents.

\section{Discussion}

To the best of our knowledge, 3 studies have investigated common mental health problems of Chinese general population during the COVID-19 epidemic (31-33). These epidemiological studies reported a wide range of prevalence rates of depressive symptoms $(21.7-48.3 \%)$ and anxiety symptoms (19.2-53.3\%), and identified some correlates of these mental health problems, including female gender, physical symptoms, and media exposure. During the initial stage of the COVID-19 pandemic in Europe, $18.7 \%$ and $21.6 \%$ of the Spanish general population had depressive and anxiety symptoms, respectively, while $32.4 \%$ and $18.7 \%$ of the Italian general population suffered from high levels of depressive and anxiety symptoms, respectively $(34,35)$. Unlike the previous studies, we investigated both the prevalence of mental health problems and patterns of mental health service use among Chinese residents, with a particular focus on subpopulation differences. Therefore, this is the first empirical study on mental health 


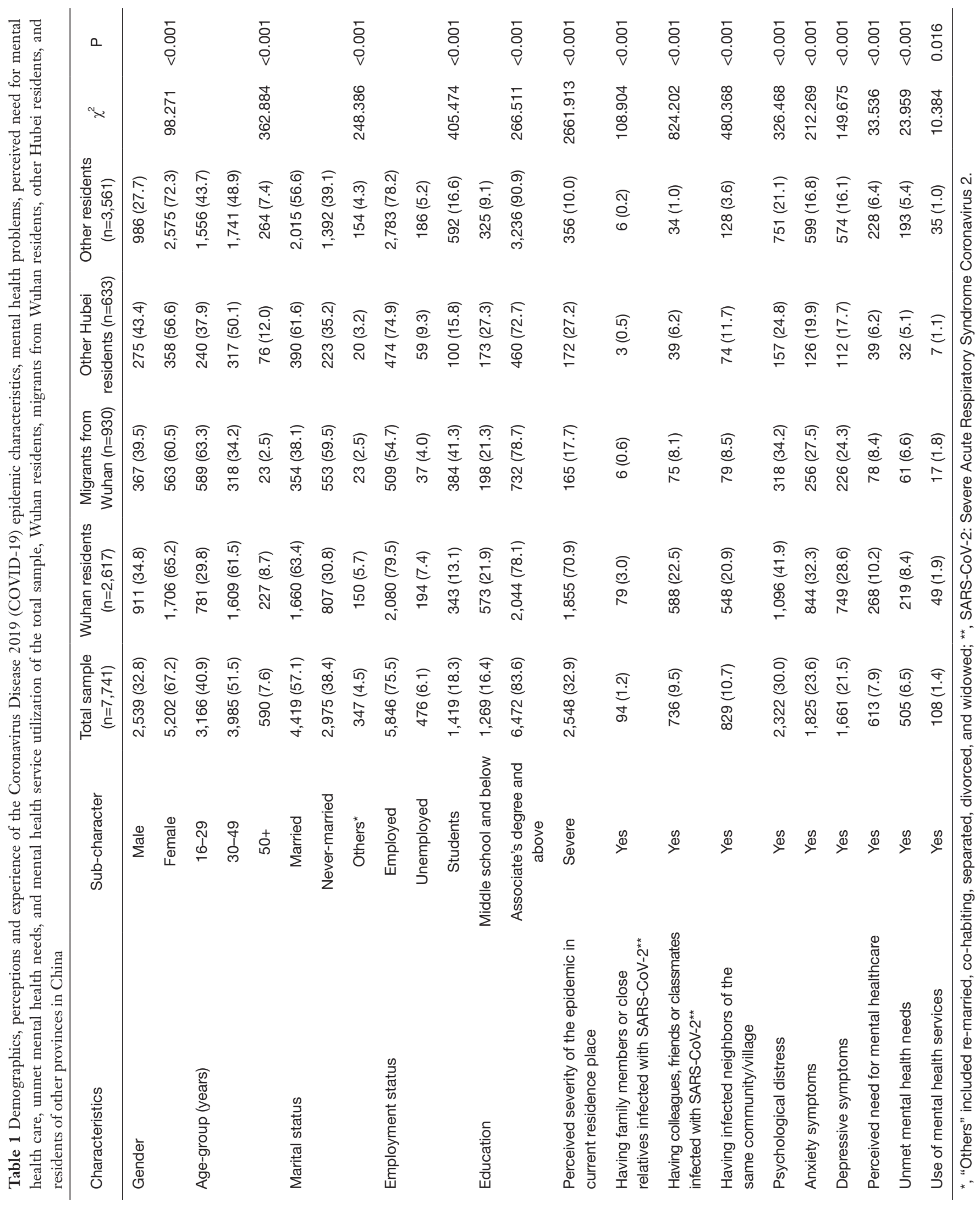


Table 2 Step-by-step multiple logistic regression analyses on relative risks of mental health problems of Wuhan residents, migrants from Wuhan, and other Hubei residents in comparison to other residents

\begin{tabular}{|c|c|c|c|c|c|c|c|}
\hline Mental health problem & Subpopulation & \multicolumn{2}{|c|}{ Step $0^{*}$} & \multicolumn{2}{|l|}{ Step $1^{\star \star}$} & \multicolumn{2}{|c|}{ Step $2^{\star \star \star}$} \\
\hline \multirow[t]{3}{*}{ Psychological distress } & Wuhan residents & $2.70(2.41,3.02)$ & $<0.001$ & $2.99(2.66,3.36)$ & $<0.001$ & $1.97(1.70,2.29)$ & $<0.001$ \\
\hline & Migrants from Wuhan & $1.94(1.66,2.28)$ & $<0.001$ & $2.33(1.97,2.94)$ & $<0.001$ & $2.04(1.73,2.41)$ & $<0.001$ \\
\hline & Other Hubei residents & $1.23(1.01,1.50)$ & 0.037 & $1.47(1.20,1.80)$ & 0.036 & $1.26(1.02,1.55)$ & 0.029 \\
\hline \multirow[t]{4}{*}{ Anxiety symptoms } & Wuhan residents & $2.35(2.09,2.65)$ & $<0.001$ & $2.59(2.29,2.94)$ & $<0.001$ & $1.79(1.52,2.10)$ & $<0.001$ \\
\hline & Migrants from Wuhan & $1.88(1.59,2.22)$ & $<0.001$ & $2.16(1.82,2.57)$ & $<0.001$ & $1.93(1.62,2.31)$ & $<0.001$ \\
\hline & Other Hubei residents & $1.23(0.99,1.52)$ & 0.059 & $1.45(1.17,1.81)$ & 0.001 & $1.27(1.02,1.59)$ & 0.036 \\
\hline & Other residents & 1 & & 1 & & 1 & \\
\hline \multirow{2}{*}{ Depressive symptoms } & Other Hubei residents & $1.12(0.90,1.40)$ & 0.324 & $1.29(1.03,1.62)$ & 0.030 & $1.13(0.89,1.42)$ & 0.311 \\
\hline & Other residents & 1 & & 1 & & 1 & \\
\hline
\end{tabular}

${ }^{*}$, no adjustment of any demographic and the Coronavirus Disease 2019 (COVID-19) epidemic related variables; ${ }^{* *}$, adjusting for demographic variables only; ${ }^{* * *}$, adjusting for demographic and COVID-19 epidemic related variables.

Table 3 Step-by-step multiple logistic regression analyses on odds of perceived need for mental health care and mental health service utilization of Wuhan residents, migrants from Wuhan, and other Hubei residents in comparison to other residents*

\begin{tabular}{|c|c|c|c|c|c|c|c|c|c|}
\hline $\begin{array}{l}\text { Mental health } \\
\text { services }\end{array}$ & Subpopulation & \multicolumn{2}{|l|}{ Step 0} & \multicolumn{2}{|l|}{ Step 1} & \multicolumn{2}{|l|}{ Step 2} & \multicolumn{2}{|l|}{ Step 3} \\
\hline \multirow[t]{3}{*}{$\begin{array}{l}\text { Perceived need for } \\
\text { mental health care }\end{array}$} & Wuhan residents & $\begin{array}{c}1.67 \\
(1.39,2.01)\end{array}$ & $<0.001$ & $\begin{array}{c}1.75 \\
(1.45,2.11)\end{array}$ & $<0.001$ & $1.33(1.04,1.70)$ & 0.025 & $0.87(0.66,1.15)$ & 0.329 \\
\hline & $\begin{array}{l}\text { Migrants from } \\
\text { Wuhan }\end{array}$ & $\begin{array}{c}1.34 \\
(1.02,1.75)\end{array}$ & 0.033 & $\begin{array}{c}1.50 \\
(1.14,1.97)\end{array}$ & 0.004 & $1.34(1.02,1.78)$ & 0.037 & $0.87(0.65,1.18)$ & 0.378 \\
\hline & Other residents & 1 & & 1 & & 1 & & 1 & \\
\hline \multirow{2}{*}{$\begin{array}{l}\text { Utilization of } \\
\text { mental health } \\
\text { services }\end{array}$} & Wuhan residents & $\begin{array}{c}1.92 \\
(1.24,2.98)\end{array}$ & 0.003 & $\begin{array}{c}1.93 \\
(1.23,3.03)\end{array}$ & 0.004 & $1.61(0.91,2.84)$ & 0.104 & $1.24(0.69,2.23)$ & 0.463 \\
\hline & Other residents & 1 & & 1 & & 1 & & 1 & \\
\hline
\end{tabular}

*, Step 0: No adjustment of any demographic and the Coronavirus Disease 2019 (COVID-19) epidemic related variables; Step 1: Adjusting for demographic variables only; Step 2: Adjusting for demographics and COVID-19 epidemic variables; Step 3: Adjusting for demographics, COVID-19 epidemic variables, and mental health problems. 
Table 4 Types of mental health services used by Chinese residents who endorsed a need for mental health care (N=108), n (\%)

\begin{tabular}{|c|c|c|c|c|c|c|}
\hline Subpopulation & Total & Face-to-face visit at an institution & Internet-based services & Telephone-based services & $\chi^{2}$ & $P$ \\
\hline Migrants from Wuhan & 17 & $7(41.2)$ & $5(29.4)$ & $5(29.4)$ & & \\
\hline Other Hubei residents & 7 & $1(14.3)$ & $3(42.9)$ & $3(42.9)$ & & \\
\hline Other residents & 35 & $21(60.0)$ & $13(37.1)$ & $1(2.9)$ & & \\
\hline
\end{tabular}

Table 5 Main reasons for not using mental health services among Chinese residents who endorsed a need for mental health care (N=505), $\mathrm{n}(\%)$

\begin{tabular}{|c|c|c|c|c|c|c|}
\hline Subpopulation & Total & Lack of availability* & Lack of accessibility & Lack of acceptability ${ }^{\&}$ & $\chi^{2}$ & $\mathrm{P}$ \\
\hline Migrants from Wuhan & 61 & $32(52.5)$ & $24(39.3)$ & $5(8.2)$ & & \\
\hline Other Hubei residents & 32 & $14(43.8)$ & $8(25.0)$ & $10(31.3)$ & & \\
\hline Other residents & 138 & $57(29.5)$ & $52(26.9)$ & $84(43.5)$ & & \\
\hline
\end{tabular}

*, Examples: 1. "I don't know where and how to find mental health specialists"; 2. "I can't seek help from them because the hospital has suspended mental health services"; 3. "There are no mental health specialists here". \#, Examples: 1. "Due to transportation restriction, I can't go to the hospital"; 2. "Because the SARS-CoV-2 is everywhere outside my home, I can't go to the hospital". 3. "The mental health services are too expensive for me". \&, Examples: 1. "I prefer to self-regulate my mood"; 2 . "Other people will discriminate me if they know I visit mental health specialists for psychological problems"; 3. "The mental health services do not work for my problems".

service utilization of the general population during the COVID-19 epidemic in China. Given that the epicenter of the COVID-19 outbreak now has shifted to major cities in Europe and North America, our findings may inform the crisis mental health care policy development and implementation in other countries affected by the global pandemic.

The main findings of this study include: first, there was increased risk of mental health problems in Wuhan residents, migrants from Wuhan, and other Hubei residents compared to other residents, for example, net of demographics, Wuhan residents, migrants from Wuhan, and other Hubei residents were 3.0-, 2.3-, and 1.5 -fold more likely to suffer from psychological distress than other residents; second, rates of unmet mental health needs among Wuhan residents and migrants from Wuhan were high, while rates of mental health service use across the 4 subpopulations were low; third, internet- and telephonebased services were more frequently used by mental health service users; fourth, the majority $(80 \%)$ of those in need did not seek help from mental health specialists due to various barriers, particularly among Wuhan residents and migrants from Wuhan.

In general population-based surveys in China using the GHQ-12, GADS-7, and PHQ-9, the prevalence rates of psychological distress, anxiety symptoms, and depressive symptoms in Chinese residents were $12.8-15.2 \%$, $7.8-9.9 \%$, and $6.2-13.4 \%$, respectively (36-40). Compared to previous prevalence estimates, we found higher prevalence of mental health problems across all subpopulations (psychological distress: $21.1-41.9 \%$, anxiety symptoms: $16.8-32.3 \%$, and depressive symptoms: 16.1-28.6\%), suggesting the increased risk of mental health problems in Chinese population affected by the COVID-19 epidemic, in particular Wuhan residents and migrants from Wuhan. In any crisis situation, the experience of fear, stress, anxiety, and uncertainty is common. Due to the effects of both the epidemic and mass quarantine to control the spread of the COVID-19, psychological distress, anxiety, and depression in the population are likely to increase. Importantly, when the study was carried out during the rapidly spreading phase of the COVID-19 epidemic, many Chinese people were stressed by the devastating effects of COVID-19 at its initial stage such as over-crowded 
hospitals, physically and emotionally exhausted physicians, shortage of protective gear, and escalating infection and death rates among the population including the health staff. The decreased ORs of Wuhan residents, migrants from Wuhan, and other Hubei residents versus other residents having mental health problems after adjusting for the COVID-19 epidemic variables suggest that the subpopulation differences in risk of mental health problems were at least in part attributed to the varying effects of the epidemic. For example, Wuhan residents had increased risk of transmission due to higher rates of infection, and also experienced greater psychological effects of stringent mass quarantine. Migrants from Wuhan, on the other hand, encountered other stressors after their departure from Wuhan such as compulsory isolation for medical observation at their destination sites, separation from their families in Wuhan, "homelessness" and inability to return to their place of origin, and social stigma and discrimination, all of which could explain the greater risk of mental health problems found in this population.

In this study, 10.2\% Wuhan residents, $8.4 \%$ migrants from Wuhan, $6.2 \%$ other Hubei residents, and $6.4 \%$ other residents reported a need for mental health care in recent days; these rates, compared to the rate of perceived mental health needs $(9.0 \%)$ among the general adults in Western Europe in the past 12 months (41), may indicate greater mental health needs among Chinese population during the epidemic. Studies have shown that mental health problems are a major determinant of subjective awareness of the need for mental health care $(41,42)$. Therefore, the significantly higher odds of perceived need for mental health care among Wuhan residents and migrants from Wuhan than other residents were likely a result of the higher prevalence of mental health problems among both subpopulations. This is also supported by the insignificant ORs of the 2 subpopulations versus the subpopulation of other residents after additional adjusting for mental health problems in the multiple analyses (Table 3).

The underutilization of mental health services is common among Chinese people in both China and Western countries (43-45). In China's eastern urban regions, where the provision of mental health services resources is relatively adequate, only $0.52-2.5 \%$ of the whole general population had ever used mental health services for their mental health problems during their lifetime $(46,47)$. In our sample, rates of mental health service use during recent days were also low, at least not proportional to the high prevalence of mental health problems and perceived need for mental health care (48): $1.0 \%$ in other residents, $1.1 \%$ in other Hubei residents, $1.8 \%$ in migrants from Wuhan, and $1.9 \%$ in Wuhan residents, suggesting that $78.6-84.4 \%$ of the 4 subpopulations in need have unmet mental health needs.

Levels of unmet mental health needs were significantly higher in Wuhan residents and migrants from Wuhan than other residents. This was mainly due to lack of accessibility and availability of mental health services during the COVID-19 epidemic, as indicated by reasons for the lack of service use in Table 5. Due to the cessation of mental health services in some overwhelmed hospitals, suspension of public transportations, and mass quarantine in Wuhan, the residents in need had little knowledge of how to access professional help and other mental health services.

Our findings on the type of mental health services used are interesting because, telephone- or internet-based services are generally less likely to be used by Chinese mental health service users $(<20 \%)$ as most Chinese users prefer face-to-face services (49). The high proportions of telephone and internet users among Wuhan residents and migrants from Wuhan indicate that help-seeking behavior and patterns was dramatically changed by the epidemic response to enforce social isolation. This was reinforced by the official recommendations to use mental health hotlines and internet-based mental health services instead of hospital-based services by the NHCC, as well as the poor availability and accessibility of conventional on-site mental health services (17).

Our study has several limitations. First, the sample of Chinese residents was recruited via social networks, and most participants were well-educated given the education level of the sample (Table 1), which limits the representativeness of our sample. Because of this, our findings may not be generalized to all Chinese residents. Second, it is not feasible to assess the presence of mental disorders in participants using clinical diagnostic interview, so objective data regarding the mental health needs of residents, as evaluated by mental health specialists, were unavailable. Third, the sample size of the subpopulation of other Hubei residents is relatively small.

\section{Conclusions}

Despite the limitations, our study provides insights into the mental health problems and service utilization of the population affected by the COVID-19 epidemic, in particular those within and outside the epicenter. In 
summary, during the period of exponential increase in the number of COVID-19 infections in China, the affected residents had an elevated risk for mental health problems and increased perceived mental health needs, in particularly Wuhan residents, migrants from Wuhan, and other Hubei residents. However, most of those in need received no mental health services due to lack of accessibility and availability. Among the limited number of mental health service users, internet- and telephone-based services were the main type of services received. Crisis mental health services should be provided during the COVID-19 epidemic, especially focusing on subpopulations at higher risk for mental health problems to address the unmet mental health needs.

Undoubtedly, the COVID-19 epidemic has put enormous pressure on the mental health care system in China. China has relatively poor mental health services which are unevenly distributed, with central China being less adequate compared to eastern China (50). Given that the COVID-19 pandemic is likely to cause unprecedented mental health burden in the affected populations globally, we urge mental health policy-makers and providers in affected countries to rapidly re-design their mental health service systems to ensure that the services are safe, flexible, and readily accessible to those in need. People who are locked down within the epicenter or have recently left the epicenter should be considered as the key target populations for the crisis mental health services. We also recommend a switch from the traditional hospital-based mental health services to internet- and telephone-based services, to remove any risk of community COVID-19 transmission and minimize treatment barriers. Although a component of hospital-based mental health services should also be provided to those with acute psychiatric risks or severe mental disorders, greater efforts are also warranted to strengthen the community mental health service system and primary care services. Finally, an integrated and coordinated service system is needed to maximize the efficiency of mental health service resources to equitably meet the needs of all affected populations.

\section{Acknowledgments}

The authors thank all participants for their cooperation and support.

Funding: The study was supported by the National Natural Science Foundation of China (71774060, Bao-Liang Zhong, PI) and Special Programs for Emergency Science and Technology Development of Novel Coronavirus Pneumonia from the Fundamental Research Funds for the Central Universities, Huazhong University of Science \& Technology (2020kfyXGYJ003, Wen-Tian Li, PI).

\section{Footnotes}

Reporting Checklist: The authors have completed the SURGE reporting checklist, available at http://dx.doi. org/10.21037/atm-20-4145

Data Sharing Statement: Available at http://dx.doi. org/10.21037/atm-20-4145.

Conflicts of Interest: All authors have completed the ICMJE uniform disclosure form (available at http://dx.doi. org/10.21037/atm-20-4145). The authors have no conflicts of interest to declare.

Ethical Statement: The authors are accountable for all aspects of the work in ensuring that questions related to the accuracy or integrity of any part of the work are appropriately investigated and resolved. The study was conducted in accordance with the Declaration of Helsinki (as revised in 2013). This study was approved by the institutional ethics board of the Ethics Committee of Wuhan Mental Health Center (No. KY2020.01.01). Informed consent was obtained from all participants. Procedures of informed consent for our online survey have been described in detail in the main text.

Open Access Statement: This is an Open Access article distributed in accordance with the Creative Commons Attribution-NonCommercial-NoDerivs 4.0 International License (CC BY-NC-ND 4.0), which permits the noncommercial replication and distribution of the article with the strict proviso that no changes or edits are made and the original work is properly cited (including links to both the formal publication through the relevant DOI and the license). See: https://creativecommons.org/licenses/by-nc-nd/4.0/.

\section{References}

1. Zhang H. Early lessons from the frontline of the 2019nCoV outbreak. Lancet 2020;395:687.

2. Yi Y, Lagniton PNP, Ye S, et al. COVID-19: what have been learned and to be learned about the novel coronavirus disease Int J Biol Sci 2020;16:1753-66. 
3. Chen S, Yang J, Yang W, et al. COVID-19 control in China during mass population movements at New Year. Lancet 2020;395:764-6.

4. World Health Organization. Coronavirus disease (COVID-2019) situation reports. 2020. Accessed June 23 2020. Available online: https://www.who.int/emergencies/ diseases/novel-coronavirus-2019/situation-reports

5. Health Commission of Hubei Province. Update of the novel coronavirus pneumonia in Hubei, April 5, 2020. Accessed April 7, 2020. Available online: http://wjw.hubei. gov.cn/fbjd/dtyw/202003/t20200330_2196392.shtml

6. World Health Organization. Report of the WHOChina Joint Mission on Coronavirus DIsease 2019 (COVID-2019). Beijing, 2020.

7. World Health Organization. 2019-nCoV outbreak is an emergency of international concern. 2020. Accessed February 16, 2020. Available online: http://www.euro. who.int/en/health-topics/emergencies/pages/news/ news/2020/01/2019-ncov-outbreak-is-an-emergency-ofinternational-concern

8. The Lancet Infectious Diseases. COVID-19, a pandemic or not? Lancet Infect Dis 2020;20:383.

9. Shigemura J, Ursano RJ, Morganstein JC, et al. Public responses to the novel 2019 coronavirus $(2019-\mathrm{nCoV})$ in Japan: Mental health consequences and target populations. Psychiatry Clin Neurosci 2020;74:281-2.

10. Person B, Sy F, Holton K, et al. Fear and stigma: the epidemic within the SARS outbreak. Emerg Infect Dis 2004;10:358-63.

11. Xu Y, Li P, Zhou J, et al. Awareness, behaviors, and mental health towards SARS of residents in Nanning. Pract Prev Med 2005;12:134-5.

12. Wang $\mathrm{P}$, Zhao M, Zhao L. Investigation of psycho-status in 293 undergraduates during and after SARS period. Chinese Journal of Health Psychology 2004;12:419-20.

13. Li A, Lin W, Zeng H. A survey on the mental health and behaviors of residents during the SARS epidemic. Chin J Public Health 2004;20:650.

14. Neria Y, Sullivan GM. Understanding the mental health effects of indirect exposure to mass trauma through the media. JAMA 2011;306:1374-5.

15. Chinazzi M, Davis JT, Ajelli M, et al. The effect of travel restrictions on the spread of the 2019 novel coronavirus (COVID-19) outbreak. Science 2020. doi: 10.1126/ science.aba9757.

16. Brooks SK, Webster RK, Smith LE, et al. The psychological impact of quarantine and how to reduce it: rapid review of the evidence. Lancet 2020. doi: 10.1016/
S0140-6736(20)30460-8.

17. Li W, Yang Y, Liu Z-H, et al. Progression of mental health services during the COVID-19 outbreak in China. Int J Biol Sci 2020;16:1732-8.

18. Xiang YT, Yang Y, Li W, et al. Timely mental health care for the 2019 novel coronavirus outbreak is urgently needed. Lancet Psychiatry 2020;7:228-9.

19. Epidemiology Working Group for NCIP Epidemic Response, Chinese Center for Disease Control and Prevention. [The epidemiological characteristics of an outbreak of 2019 novel coronavirus diseases (COVID-19) in China]. Zhonghua Liu Xing Bing Xue Za Zhi 2020;41:145-51.

20. Furukawa TA, Kessler RC, Slade T, et al. The performance of the K6 and K10 screening scales for psychological distress in the Australian National Survey of Mental Health and Well-Being. Psychol Med 2003;33:357-62.

21. Mamun MA, Hossain MS, Moonajilin MS, et al. Does loneliness, self-esteem and psychological distress correlate with problematic internet use? A Bangladeshi survey study. Asia Pac Psychiatry 2020;12:e12386.

22. Yang T, Huang L, Wu Z. Study on the appropriateness of the Chinese version of the General Health Questionnaire as a screening instrument for psychological disorders in mainland China. Zhonghua Liu Xing Bing Xue Za Zhi 2003;24:769-73.

23. Zhong BL, Liu TB, Chan SS, et al. Common mental health problems in rural-to-urban migrant workers in Shenzhen, China: prevalence and risk factors. Epidemiol Psychiatr Sci 2018;27:256-65.

24. Zhou Y, Bi Y, Lao L, et al. Application of GAD-7 in population screening for generalized anxiety disorder. Chinese Journal of General Practitioners 2018;17:735-7.

25. Wang W, Bian Q, Zhao Y, et al. Reliability and validity of the Chinese version of the Patient Health Questionnaire (PHQ-9) in the general population. Gen Hosp Psychiatry 2014;36:539-44.

26. Leung DYP, Mak YW, Leung SF, et al. Measurement invariances of the PHQ-9 across gender and age groups in Chinese adolescents. Asia Pac Psychiatry 2020;12:e12381.

27. Zeng Q, He Y, Liu H, et al. Reliability and validity of Chinese version of the Generalized Anxiety Disorder 7-item (GAD-7) scale in screening anxiety disorders in outpatients from traditional Chinese internal department. Chin Ment Health J 2013;27:163-8.

28. Sun XY, Li YX, Yu CQ, et al. Reliability and validity of depression scales of Chinese version: a systematic review. Zhonghua Liu Xing Bing Xue Za Zhi 2017;38:110-6. 
29. Nelson CH, Park J. The nature and correlates of unmet health care needs in Ontario, Canada. Soc Sci Med 2006;62:2291-300.

30. Lee S, Guo WJ, Tsang A, et al. Perceived barriers to mental health treatment in metropolitan China. Psychiatr Serv 2010;61:1260-2.

31. Wang C, Pan R, Wan X, et al. Immediate Psychological Responses and Associated Factors during the Initial Stage of the 2019 Coronavirus Disease (COVID-19) Epidemic among the General Population in China. Int J Environ Res Public Health 2020;17:1729.

32. Wang Y, Gao J, Chen H, et al. The relationship between media exposure and mental health problems during COVID-19 outbreak. Fudan University Journal of Medical Sciences 2020;47:174-9.

33. Li Y, Wang X, Zhang J, et al. Mental health survey among Chinese general public during the COVID-19 epidemic. International Infectious Diseases (Electronic Edition) 2020;9:308-10.

34. González-Sanguino C, Ausín B, Castellanos MÁ, et al. Mental health consequences during the initial stage of the 2020 Coronavirus pandemic (COVID-19) in Spain. Brain Behav Immun 2020;87:172-6.

35. Mazza C, Ricci E, Biondi S, et al. A Nationwide Survey of Psychological Distress among Italian People during the COVID-19 Pandemic: Immediate Psychological Responses and Associated Factors. Int J Environ Res Public Health 2020;17:3165.

36. Liu H, Zhu Z. A GHQ investigation on the mental health of workers of a mega-size state-owned enterprise of Shanghai. China Medical Herald 2011;8:144-6.

37. Yuan J, Zhu W, Li X, et al. A GHQ investigation on the mental health of 2749 community residents. Shanghai Arch Psychiatry 2005;17:27-8.

38. Qi X. Emotional problems and service needs of community residents. Shanghai: Shanghai Jiaotong University, 2014.

39. Sun J, Luo Y, Zhang R. Relationship of depression and anxiety with cognitive emotion regulation strategies among the elderly in the community. Chinese General Practice

Cite this article as: Zhong BL, Zhou DY, He MF, Li Y, Li WT, Ng CH, Xiang YT, Chiu HFK. Mental health problems, needs, and service use among people living within and outside Wuhan during the COVID-19 epidemic in China. Ann Transl Med 2020;8(21):1392. doi: 10.21037/atm-20-4145
2019;22:3887-92.

40. Tu Q, Han A, Qin Y, et al. Prevalence of depression in adult residents and its influence factors in Jiangsu province. Modern Med J 2018;46:1205-9.

41. Codony M, Alonso J, Almansa J, et al. Perceived need for mental health care and service use among adults in Western Europe: results of the ESEMeD project. Psychiatr Serv 2009;60:1051-8.

42. Villatoro AP, Mays VM, Ponce NA, et al. Perceived Need for Mental Health Care: The Intersection of Race, Ethnicity, Gender, and Socioeconomic Status. Soc Ment Health 2018;8:1-24.

43. Phillips MR, Zhang J, Shi Q, et al. Prevalence, treatment, and associated disability of mental disorders in four provinces in China during 2001-05: an epidemiological survey. Lancet 2009;373:2041-53.

44. Abe-Kim J, Takeuchi DT, Hong S, et al. Use of mental health-related services among immigrant and US-born Asian Americans: results from the National Latino and Asian American Study. Am J Public Health 2007;97:91-8.

45. Kudva KG, El Hayek S, Gupta AK, et al. Stigma in mental illness: Perspective from eight Asian nations. Asia Pac Psychiatry 2020;12:e12380.

46. Zhou J. The survey of mental health service utilization in Xiamen city. China Modern Med 2016;23:170-2.

47. Wei Z, Liu T, Hu C, et al. The mental health service utilization in Shenzhen city. Chin Ment Health J 2010;24:597-603.

48. Zhao K, He Y, Zeng Q, et al. Factors of Mental Health Service Utilization by Community-Dwelling Adults in Shanghai, China. Community Ment Health J 2019;55:161-7.

49. Zhang Y, Li Y, Chen Q, et al. Intent of help-seeking and health service utilization research of patients with mental disorder in Dalian. Sichuan Mental Health 2016;29:184-6.

50. Zhong BL, Ruan YF, Xu YM, et al. Prevalence and recognition of depressive disorders among Chinese older adults receiving primary care: A multi-center crosssectional study. J Affect Disord 2020;260:26-31. 